\title{
Micro- and Nucleolar-organizing B-Chromosomes in Calycadenia (Asteraceae)
}

\author{
Gerald D. Carr and Robert L. Carr \\ Department of Botany, University of Hawaii, 3190 Maile Way, Honolulu, Hawaii \\ 96822 U.S.A. and Department of Biology, Eastern Washington University, \\ 260 Hall of Sciences, Cheney, Washington 99004 U.S.A.
}

Received January 6, 1980

Recent reviewers of B-chromosomes in plants and animals (Battaglia 1964, Jones 1975) concur that examples of nucleolar organization by B-chromosomes are extremely rare, perhaps limited to one plant and one insect. Biosystematic studies in the genus Calycadenia (Carr 1975a, b, 1977) have led to the discovery of at least three recognizable forms of nucleolar-organizing B-chromosomes, one type in each of five different taxa embracing three species of the genus. Another kind of B-chromosome, unusual because of its extremely small size (less than $1 \mu$ ), was also found in a species of Calycadenia. The characterization of the several types of B-chromosomes found in Calycadenia is the purpose of this paper.

Calycadenia is an annual, predominantly self-incompatible genus in the tarweed subtribe of the Heliantheae (Asteraceae). It is comprised of about a dozen species confined mostly to arid regions bordering the Central Valley of California. At least two of the species consist of several chromosome races differentiated chiefly by translocations and in one instance also by aneuploid reduction (Carr 1975a, 1977). An additional cytological peculiarity of Calycadenia is the fact that most, if not all species in the genus have two pairs of nucleolar-organizing chromosomes despite the fact that they are diploids with low chromosome numbers $(n=4,5,6,7$ and 9; Carr 1975a).

\section{Materials and methods}

Observation of meiosis in microsporocytes provided the information on occurrence, behavior and morphology of B-chromosomes presented herein. Some of the floral bud material examined in this study was collected directly from natural populations (Table 1). Fruits were also collected directly from natural populations (Table 1), from which plants were grown under greenhouse conditions for use in a hybridization program (see Carr 1975a for details). These parental and subsequent hybrid greenhouse-grown plants provided the balance of the material used in this study. Fixation, slide preparation and estimation of pollen fertility were accomplished as described elsewhere (Carr 1975a). 
Table 1. Populations of Calycadenia in which

B-chromosomes have been observed ${ }^{\dagger}$

C. ciliosa Greene race Dry Creek

663 Tehama Co., $6.0 \mathrm{mi}$ W of Vestal Rd. on Hwy. 36, $n=6^{*}$

C. multiglandulosa DC. ssp. cephalotes (DC.) Keck

566 Napa Co., 1.5 mi SW of Angwin on Howell Mt. Rd., $n=6^{*}$

577 Sonoma Co., Jct. of Facienda Lane and Occidental Rd., $n=6^{*}$

639 Marin Co., E city limits of Forest Knolls, $n=6^{*}$

C. multiglandulosa ssp. robusta Keck

627 Santa Clara Co., $3.3 \mathrm{mi}$ NE of Skyline Blvd. on Page Mill Rd., $n=6 *,+\dagger$

630 Santa Clara Co., $1.2 \mathrm{mi}$ SW of San Martin, $n=6^{*}$

C. oppositifolia (Greene) Greene

789 Butte Co., Upper Bidwell Park, near Chico, $n=7^{* *}$

C. pauciflora Gray race Pauciflora

606 Colusa Co., $0.5 \mathrm{mi}$ NW of Fout Spr. Boys Ranch, $\mathrm{n}=5^{*}$

645 Tehama Co., $8.0 \mathrm{mi}$ SW of Paskenta on rd. to Mud Flat Campground, $\mathrm{n}=5^{*}$

646 Tehama Co., 7.1 mi NW of Paskenta on Toomes Camp Rd., $n=5^{*}$

652 Colusa Co., $3.6 \mathrm{mi} \mathrm{SW}$ of Leesville on Bartlett Spr. Rd., $n=5^{*}$

671 Lake Co., $2,2 \mathrm{mi}$ SW of Rieff Rd. on Morgan Valley Rd., $\mathrm{n}=5^{*}$

C. pauciflora race Ramulosa

680 Lake Co., SW Lakeport, $n=6^{*}$

C. pauciflora race Tehama

738 Tehama Co., $0.1 \mathrm{mi} \mathrm{S}$ of Hwy. 36 on Tedoc Rd., $\mathrm{n}=6^{*}$

743 Tehama Co., $1.4 \mathrm{mi} \mathrm{W}$ of Mountain Rd, on Colyer Spr. Rd., $\mathrm{n}=6^{*}$

+ All populations are located in the state of California (U.S.A.) and all collection numbers are those of the senior author.

* Nucleolar-organizing B's also present.

\# Micro-B's also present.

** Marco-B's also present.

\section{Results}

\section{Nucleolar-organizing B-chromosomes}

Nucleolar-organizing B-chromosomes were found in a total of 14 populations (Table 1) comprising six taxa of three species of Calycadenia. At least three different types could be distinguished on the basis of size. The morphology and behavior of nucleolar-organizing B-chromosomes are described systematically below.

Calycadenia multiglandulosa ssp. robusta: In two populations of this taxon individuals with 0-3 nucleolar-organizing B-chromosomes about $3 \mu$ in length were detected (Table 1; Fig. 1). The nucleolar-organizing function of this B-chromosome when present in single dose is very strong and it can be seen associated with the nucleolus in nearly $100 \%$ of the microsporocytes, often to the exclusion of either of the two A-chromosome nucleolar-organizing pairs. When two or more Bchromosomes are present the ratio of $\mathrm{A}$ - to B-chromosomes associated with the nucleolus increases. The B-chromosomes in this taxon may pair with each other when more than one is present (cf. B's in ssp. cephalotes, Fig. 4), but they appear incapable of true pairing with any of the A-chromosomes. During the first meiotic division univalent B-chromosomes generally precede the A-chromosomes to the poles in random fashion without dividing equationally. Equational division of $\mathrm{B}$ - 
chromosomes in this and the next two taxa occurs almost always at the second meiotic division, but rarely may occur at the first anaphase. At the second meiotic metaphase the B-chromosome appears submetacentric and obviously smaller than the A-chromosomes (Fig. 2, right inset).

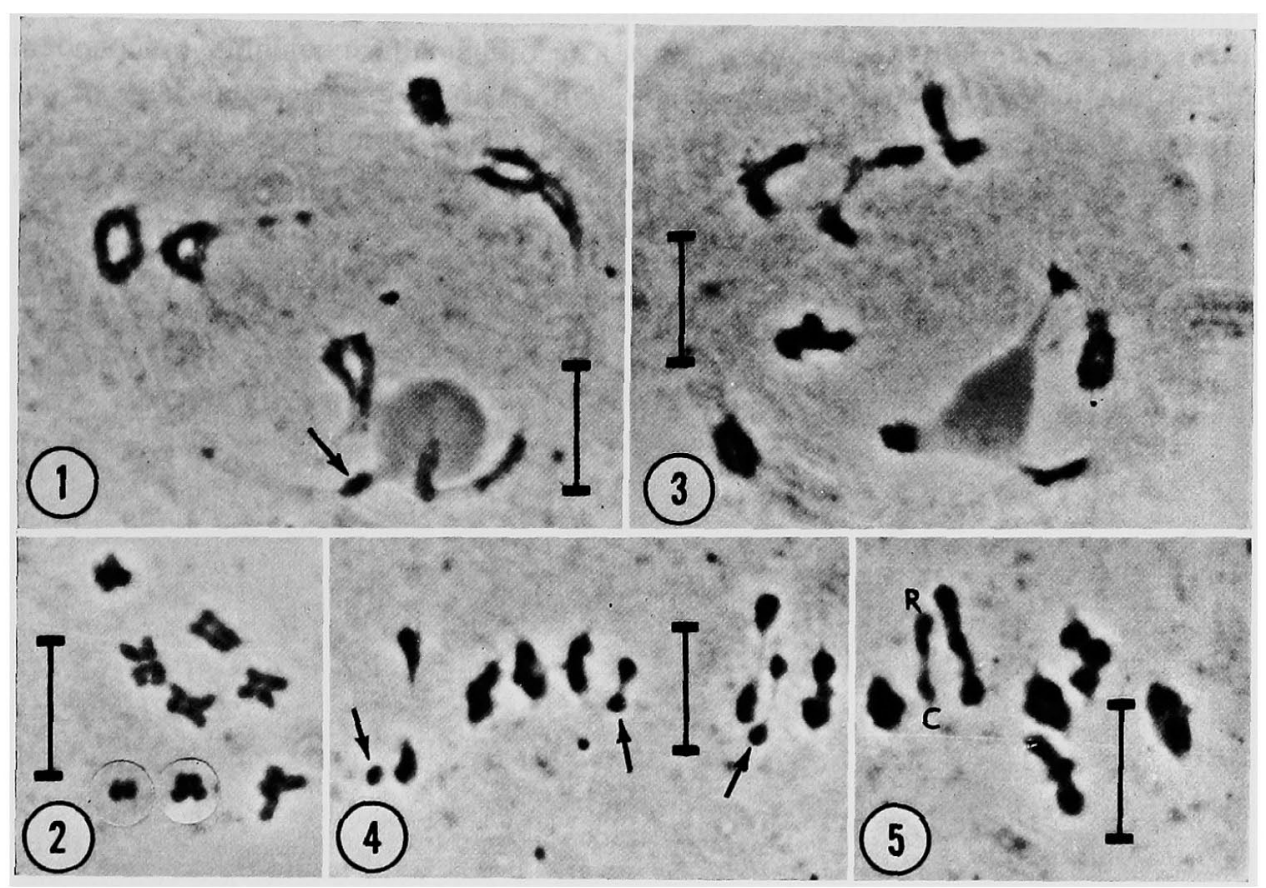

Figs. 1-5. Meiotic behavior of nucleolar-organizing B-chromosomes in Calycadenia multiglandulosa. 1, ssp. robusta, one $B_{I}$ organizing the nucleolus (arrow) $+6_{I I}$ at diakinesis. 2 , ssp. robusta $\times$ ssp. cephalotes, composite showing "cephalotes" B (left inset) and "robusta" B (right inset) with A karyotype, all at metaphase two. 3, ssp. cephalotes, two $\mathrm{B}_{11}$ +one $\mathrm{B}_{\mathrm{I}}$ organizing the nucleolus $+6_{I I}$ at diakinesis. 4, ssp. cephalotes, two $B_{1 I}+$ one $B_{I}$ (arrow) $+6_{I I}$ at metaphase one. 5 , ssp. robusta $\times$ ssp. cephalotes, $6_{11}+$ "cephalotes" B (C) and "robusta" B (R) paired at metaphase one. (Bars $=10 \mu)$.

Calycadenia multiglandulosa ssp. cephalotes: In three populations of this taxon individuals with 0-5 nucleolar-organizing B-chromosomes about $2 \mu$ in length were detected (Table 1; Fig. 3). It is possible to compare the morphology of this B-chromosome directly with that of the one described above in the hybrid between C. multiglandulosa ssp. robusta and ssp. cephalotes (Fig. 2, left and right insets; cf. Carr 1977, Fig. 3). The B-chromosome in ssp. cephalotes is smaller than the one in ssp. robusta and is apparently nearly acrocentric. The frequency of nucleolar organization by the B-chromosome from ssp. cephalotes is about equivalent to that of ssp. robusta and the meiotic behavior is also the same. Although these B-chromosomes tend to be sticky they apparently do not truly pair with the Achromosomes. They appear to pair normally with one another (Figs. 3, 4) and the "cephalotes" type also occasionally pairs with the "robusta" type in the hybrid between the subspecies (Fig. 5).

Calycadenia pauciflora race Ramulosa: In one population of this taxon 
individuals with $0-3$ nucleolar-organizing B-chromosomes about $2.5 \mu$ in length were detected (Table 1; Fig. 6). The nucleolar-organizing function of this Bchromosome is hard to detect, especially in individuals with more than one Bchromosome present. In some individuals with a single B-chromosome, the Borganizer is associated with the nucleolus in about one out of four microsporocytes. It can also occasionally be seen to organize a second smaller nucleolus, independent of the one organized by the A-pairs (Fig. 6). Although occasional associations between nucleolar-organizing pairs can appear rather strong, they are probably due to stickiness rather than true pairing. "Stickiness" in the sense used herein

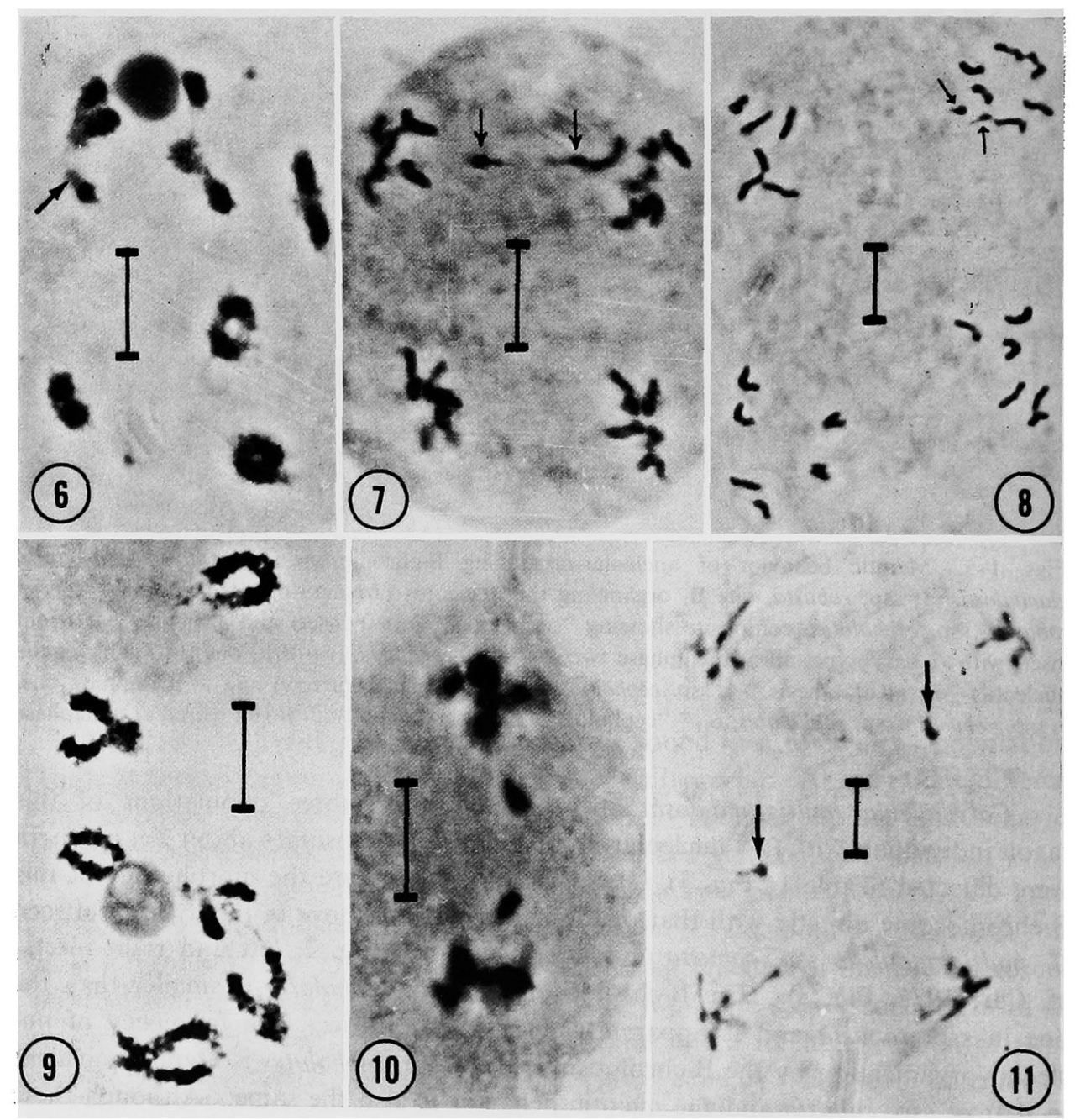

Figs. 6-11. Meiotic behavior of nucleolar-organizing B-chromosomes in Calycadenia pauciflora. Figs. 6-8. Race Ramulosa. 6, one $B_{I}$ organizing small nucleolus (arrow) $+6_{I I}$ at diakinesis. 7, late separation of B-chromatids (arrow) at telophase two. 8, two B-chromatids (arrows) incorporated in one pole at telephase two. 9-11, race Pauciflora. 9, $\mathrm{B}_{\mathrm{II}}$ organizing nucleolus $+5_{\mathrm{II}}$ at diakinesis. 10, lagging B-chromatids after equational division at telophase one. 11, two Bchromatids (arrows) excluded from the poles at telophase two. (Bars $=10 \mu$ ). 
refers to apparent achiasmate associations between chromosome segments that are chiefly heterochromatic, especially nucleolar-organizing regions or arms. Associations of this sort appear to result from the physical tackiness of the chromosomal material rather than from structural homology. Even the rather infrequent associations between B-chromosomes in this taxon usually appear to be the result of stickiness. The meiotic behavior of the B-chromosomes in this taxon is the same as in the preceding cases except that during equational division at the second meiotic metaphase the chromatids of the B-chromosome separate late, often lagging on the metaphase plate (Fig. 7). The late separation of the B-chromatids may result in their exclusion from the A-chromosome nuclei or alternatively sometimes results in the inclusion of both B-chromatids in the same microcyte nucleus (Fig. 8).

Calycadenia pauciflora race Pauciflora: In 5 populations of this taxon individuals with 0-4 large nucleolar-organizing B-chromosomes about $5 \mu$ in length were detected (Table 1; Fig. 9). The strength of nucleolar organization of this B-chromosome when present as a univalent often exceeds that of both A-nucleolarorganizing pairs. As in the previous case there are infrequent associations between the B-chromosome of this taxon and the nucleolar-organizing arms of the Achromosomes but these are probably due to stickiness of nucleolar organizers rather than true chromosomal homology. When two B-chromosomes are present they are nearly always paired with each other (cf. Fig. 9). Equational division of univalent B-chromosomes from race Pauciflora occurs most frequently at the second meiotic anaphase following their random movement to the poles of the dyad. However, about $20 \%$ of the time equational division occurs during the first meiotic division but lags behind that of the A-chromosomes (Fig. 10). As a result the Bchromatids are often excluded from the microcytes, or at least from their A-chromosome nuclei (Fig. 11).

These B-chromosomes appear to have a significant effect on the fertility of the plants possessing them. Nine controlled interpopulational hybrid individuals of race Pauciflora with one B-nucleolar-organizing chromosome apiece averaged $86 \%$ pollen stainability whereas two hybrids with two B-chromosomes apiece averaged $64 \%$ pollen stainability and one hybrid with three B's had $24 \%$ stainable pollen. Thirty-five such hybrids lacking B-chromosomes had a mean pollen stainability of $93 \%$

Calycadenia pauciflora race Tehama: In two populations of this taxon individuals with 0-2 large B-chromosomes similar to those seen in race Pauciflora were detected. A small sample of microsporocytes suggests the nucleolar-organizing function of these B-chromosomes (Fig. 12), but more material is needed to further document this function and to make observations on their meiotic behavior.

Calycadenia ciliosa race Dry Creek: The nucleolar-organizing function of a large B-chromosome from a population of this taxon is strongly suggested by observations of a small number of microsporocytes (Fig. 13). More material is needed for further substantiation and to characterize the meiotic behavior of this Bchromosome. 

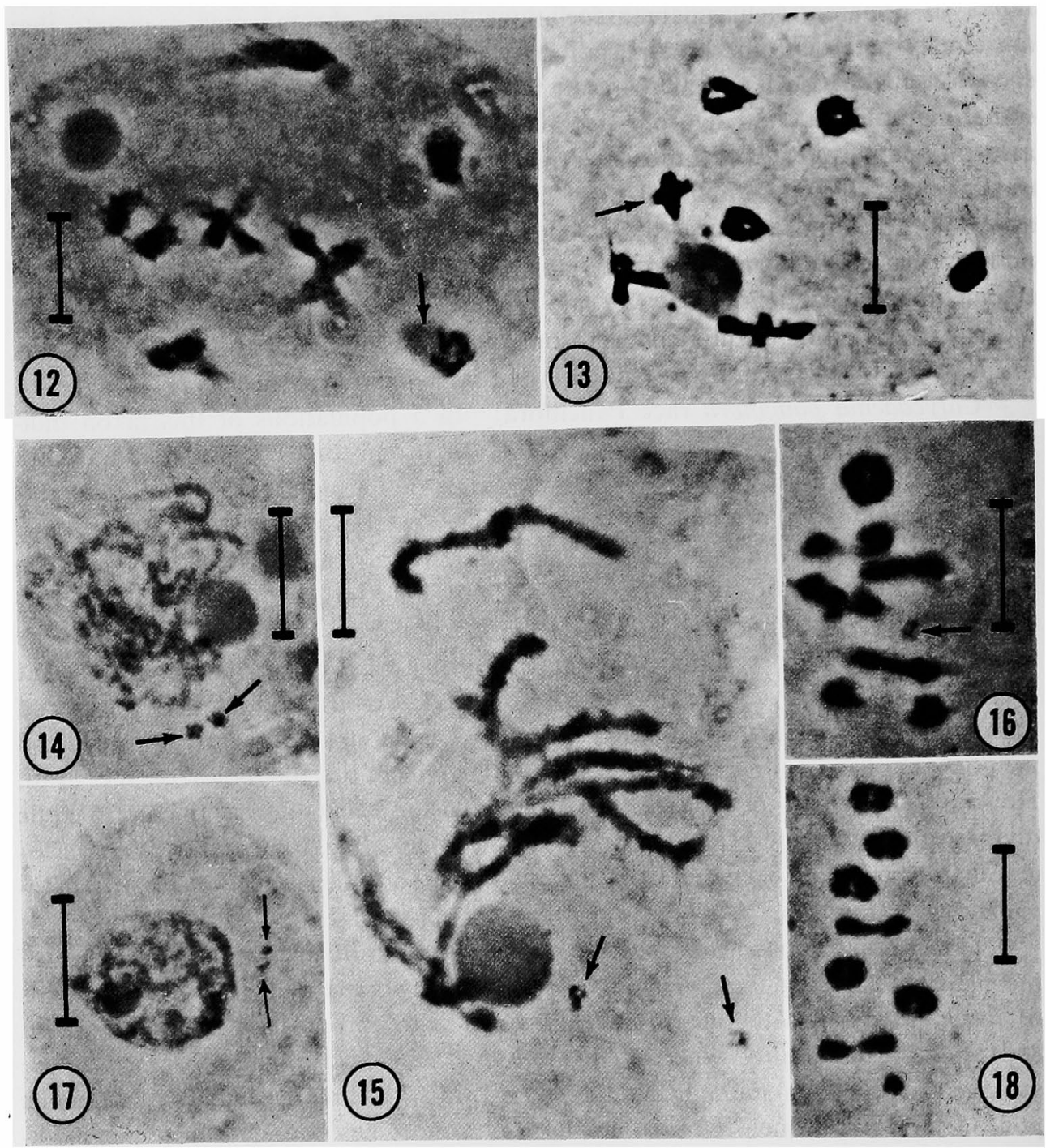

Figs. 12-18. 12, 13: Meiotic behavior of nucleolar-organizing B-chromosomes in Calycadenia. 12 , C. pauciflora race Tehama, $B_{\mathrm{II}}$ organizing one of three nucleoli (arrow) $+6_{\mathrm{II}}$ at diakinesis. 13, C. ciliosa race Dry Creek, $\mathrm{B}_{\mathrm{II}}$ organizing nucleolus (arrow) $+6_{\mathrm{II}}$ at diakinesis. (Bars $=10 \mu$ ). 14-18: Micro- and macro-B-chromosomes in Calycadenia. Figs. 14-17. Meiotic behavior of micro-B-chromosomes in Calycadenia multiglandulosa ssp. robusta. 14, two clusters of micro-B's (arrows) at early prophase. 15, micro-B's (arrows) $+6_{\mathrm{II}}$ at diplonema-diakinesis. 16, micro-B (arrow) $+6_{\text {II }}$ at metaphase one, note terminal chromomeres. 17, micro-B's (arrows) excluded from nucleus of microcyte. $18, C$. oppositifolia, one $B_{I}+7_{I I}$ at metaphase one. $(B a r s=10 \mu)$.

\section{Micro-B-chromosomes}

Microsporocytes in some individuals of one population of Calycadenia multiglandulosa ssp. robusta were found to contain very small structures less than $1 \mu$ in length that are interpreted here as micro-B-chromosomes (cf. Carter and SmithWhite 1972). These micro-B-chromosomes appear to be unstable in the micro- 
sporocytes of a single plant with numbers ranging from perhaps 0 to about 10 . Their small size and irregular clumping (Figs. 14-16) make accurate determinations difficult. In one cell at least seven micro-B's were clustered tightly but irregularly together. The condensation and staining of these structures varied little from early prophase of meiosis to the formation of microspores (Figs. 14-17). In all cells at all stages where critical observations were possible the micro-B's appeared to be double stranded, each strand possessing a light-staining proximal region with a single darker staining chromomere at each end (Fig. 16). No form of division of micro-B-chromosomes could be substantiated at any stage of meiosis of the A-complement. In some cells the micro-B-structures appeared to be of approximately the same width as a pachytene A-pair of chromosomes.

\section{Macro-B-chromosome}

A B-chromosome of dimensions only slightly smaller than chromosomes of the A-complement has been detected in one population of Calycadenia oppositifolia (Fig. 18). This is the only known occurrence of B-chromosomes in a 7-paired species of Calycadenia. The sample of microsporocytes available did not contain stages necessary to detect the potential nucleolar-organizing function of this Bchromosome, nor to characterize its meiotic behavior.

\section{Discussion}

\section{Nucleolar-organizing $B$ 's}

In view of the near absence of reports of nucleolar-organizing B-chromosomes in the literature (cf. Battaglia 1964, Jones 1975) it is peculiar for them to be so common in Calycadenia. The occurrence in Calycadenia of two nucleolar-organizing pairs of A-chromosomes is also apparently rare or at least unusual in diploid species. Some species of Calycadenia are chromosomally very active to the extent that they are characterized by a series of populations differentiated from one another by reciprocal chromosome translocations (Carr 1975a). Furthermore, these translocations often involve nucleolar-organizing chromosomes. Thus, it is tempting to speculate an origin of nucleolar-organizing B's from products of abnormal meiosis in complex structural heterozygotes produced by hybridizing species or populations. The apparent lack of chromosomal differentiation within Calycadenia multiglandulosa (Carr 1977) would in this case appear to necessitate interspecific hybridization to produce the effects outlined above.

Alternatively, it is perhaps equally probable that the aneuploidy common in Calycadenia has been involved in the production of the nucleolar-organizing B's by supplying a centromeric fragment onto which a nucleolar-organizing region could be readily translocated. The presence of four nucleolar-organizing chromosomes in the basic set increases the possibility of this occurrence.

The nucleolar-organizing B-chromosomes in Calycadenia are obviously not all identical and it seems probable that they have had more than one origin. Those of $C$. multiglandulosa ssp. robusta and ssp. cephalotes, although slightly different in size and morphology are partially homologous as indicated by apparent chi- 
asmate pairing in artificial hybrids between the subspecies. The two types quite probably had a common origin and subsequently became differentiated through gain or loss of chromosomal material. Several such modifications of B-chromosomes in Aster ageratoides have been described in detail by Matsuda (1970). Calycadenia pauciflora races Tehama and Pauciflora and $C$. ciliosa have nucleolarorganizing B's that are similar to each other in size and appearance, but differ in these features from those of $C$. multiglandulosa. Considering these facts and the overall genetic relationships of these groups (Carr 1977), a common origin for the B-chromosomes of the former three taxa that is independent from the origin of the B-chromosomes in C. multiglandulosa is suspected. For the same reasons it seems probable that the B-chromosomes in C. pauciflora race Ramulosa were independently derived.

Except for the nucleolar-organizing function, the meiotic behavior of the B-chromosomes reported on here is in line with that reported in many other species (cf. Battaglia 1964, Jones 1975). Likewise, the reduction of fertility in plants with increased numbers of nucleolar-organizing B's seen in Calycadenia pauciflora race Pauciflora is in agreement with many other reports on the effects of B-chromosomes in general.

\section{Micro-B's}

The diminutive size of the structures referred to herein as micro-B's make them difficult to characterize with certainty. Other workers have reported B-chromosomes of similar dimensions (eg. Carter and Smith-White 1972), but the intensity of staining, the morphology and the behavior in the instances known to us are more typical of larger B's than the micro-B's reported here. Although division of the micro-B's in microsporocytes of Calycadenia could not be substantiated, their small size and the difficulty of observation in the material available precludes any firm conclusion in this regard. The morphology of the micro-B's repeatedly seen in many cells is remarkably similar to that of the centromeric regions of the chromosomes of Hyacinthus orientalis and Secale cereale as illustrated by Limade-Faria (1956, Figs. 6 and 9, respectively). The possible occurrence of "naked" centromeres in natural plant populations would be of considerable interest to students of chromosome evolution, but more definitive evidence beyond the scope of this report is needed to make such a claim about the micro-B's in Calycadenia.

Macro-B's

The macro-B's of one population of Calycadenia oppositifolia, although only slightly smaller than A-chromosomes of that species, are no larger than some of the nucleolar-organizing B-chromosomes in other species of Calycadenia. In fact, they are about the same size as those found in C. pauciflora races Tehama and Paucifora and $C$. ciliosa race Dry Creek. When more material from this population of $C$. oppositifolia is available it may demonstrate a nucleolar-organizing function for this B-chromosome as well. As $\mathrm{n}=7$ is considered basic in Calycadenia (Carr 1975), the occurrence of B-chromosomes of any sort in a 7-paired species like Calycadenia oppositifolia presumably indicates an origin through some means 
other than as a remnant of aneuploid reduction.

\section{Acknowledgements}

We would like to thank Dr. Donald W. Kyhos for his helpful comments on an early draft of this paper.

\section{Summary}

Cytological studies of seven taxa in four species of the genus Calycadenia revealed the existence of several types of B-chromosomes. Of special interest is the occurrence of at least three forms of nucleolar-organizing B's in a total of six taxa in three species of the genus. Also of note is the occurrence in one species of an unusual structure less than $1 \mu$ in length that is interpreted as a micro-B chromosome. Its morphology is reminiscent of that of a "naked" centromere. The Bchromosomes of Calycadenia are variable in size, morphology, and meiotic behavior. They have probably had multiple origins, perhaps as abnormal meiotic products in structural heterozygotes produced by hybridizing populations or species or as by-products of aneuploid reduction.

\section{Literature cited}

Battaglia, E. 1964. Cytogenetics of B-chromosomes. Caryologia 17: 245-299.

Carr, G. D. 1975 a. Chromosome evolution and aneuploid reduction in Calycadenia pauciflora (Asteraceae). Evolution 29: 681-699.

- 1975b. Calycadenia hooveri (Asteraceae), a new tarweed from California. Brittonia 27: 136-141.

- 1977. A cytological conspectus of the genus Calycadenia (Asteraceae): An example of contrasting modes of evolution. Amer. J. Bot. 64: 694-703.

Carter, C. R. and Smith-White, S. 1972. The cytology of Brachycome lineariloba III. Accessory chromosomes. Chromosoma 39 : 361-379.

Jones, R. N. 1975. B-chromosome systems in flowering plants and animal species. Int. Rev. Cytol. 40: 1-100.

Lima-de-Faria, A. 1956. The role of the kinetochore in chromosome organization. Hereditas 42: 85-160.

Matsuda, T. 1970. On the accessory chromosomes of Aster I. The accessory chromosomes of Aster ageratoides group. J. Sci. Hiroshima Univ., Ser. B. Div. 13: 1-63. 